\title{
Sarcopenia defined by psoas muscle index independently predicts long-term survival after living donor liver transplantation in male recipients
}

\author{
Yifei Tan ${ }^{1 \#}$, Ting Duan ${ }^{2 \#}$, Bo Li ${ }^{1}$, Bohan Zhang ${ }^{1}$, Yunfeng Zhu ${ }^{1}$, Ke Yan ${ }^{3}$, Jiulin Song ${ }^{1}$, Tao Lv $^{1}$, \\ Jian Yang ${ }^{1}$, Li Jiang ${ }^{1}$, Jiayin Yang ${ }^{1}$, Tianfu Wen ${ }^{1}$, Lunan Yan ${ }^{1}$ \\ ${ }^{1}$ Liver Transplantation Center, Department of Liver Surgery, West China Hospital of Sichuan University, Chengdu, China; ${ }^{2}$ Department of \\ Radiology, West China Hospital of Sichuan University, Chengdu, China; ${ }^{3}$ West China School of Public Health, Sichuan University, Chengdu, China
}

Contributions: (I) Conception and design: J Yang, T Wen, L Yan; (II) Administrative support: T Lv, J Yang, L Jiang; (III) Provision of study materials or patients: J Song, J Yang; (IV) Collection and assembly of data: Y Tan, T Duan, B Li, B Zhang; (V) Data analysis and interpretation:Y Tan, T Duan, Y Zhu, K Yan; (VI) Manuscript writing: All authors; (VII) Final approval of manuscript: All authors.

\#These authors contributed equally to this work.

Correspondence to: Jiayin Yang. West China Hospital of Sichuan University, Chengdu 610041, China. Email: doctoryjy@scu.edu.cn.

Background: The effect of sarcopenia on long-term outcomes in recipients after living donor liver transplantation (LDLT), including overall survival and hepatocellular carcinoma (HCC) recurrence, remains unclear, especially in China.

Methods: From 2009 to 2015, 117 adult patients underwent LDLT in our center. In all, 82 patients who had computed tomography images reaching the third lumbar vertebra level within 1 month of LDLT were included; 70 male patients were included in the final analysis after excluding 12 female patients because of poor performance of the calculated cutoff value. Sarcopenia was defined according to the psoas muscle index (PMI) cutoff value, which was calculated based on dynamic time-dependent outcomes using X-tile software. Cox proportional hazards models were used to assess multivariate-adjusted hazards ratios (HRs) to seek potential correlations between sarcopenia and posttransplant outcomes.

Results: According to the cutoff value of PMI $\left(6.25 \mathrm{~cm}^{2} / \mathrm{m}^{2}\right), 38$ patients $(54.3 \%)$ were diagnosed with sarcopenia. After an average of 63.3 months of follow-up, 21 patients died after LDLT, 16 in the sarcopenia group and 5 in the non-sarcopenia group, respectively. Sarcopenia was identified as being significantly associated with worse posttransplant overall survival in multivariate analysis, resulting in an HR of 3.22 [95\% confidence interval (CI), 1.15-8.98]. Among the 50 recipients with HCC, sarcopenia was significantly associated with HCC recurrence in univariate analysis (HR 2.87, 95\% CI, 1.06-7.80) but was not detected as an independent risk factor of HCC recurrence in multivariate analysis, although a trend (tendency)towards significance was observed (HR 2.60, 95\% CI, 0.95-7.10; $\mathrm{P}=0.062$ ).

Conclusions: Sarcopenia defined by PMI is a feasible and reliable independent predictor of posttransplant overall survival in male LDLT candidates. However, its correlation with posttransplant HCC recurrence remains uncertain.

Keywords: Sarcopenia; psoas muscle index (PMI); cutoff value; living donor liver transplantation (LDLT)

Submitted Mar 21, 2021. Accepted for publication Jun 25, 2021.

doi: 10.21037/qims-21-314

View this article at: https://dx.doi.org/10.21037/qims-21-314

\footnotetext{
^ ORCID: 0000-0001-8168-3971.
} 


\section{Introduction}

Liver transplantation (LT) is regarded as the only potential curative therapy for end-stage liver disease and selected liver cancers. However, the severe shortage of donor livers leads to an unsatisfactory mortality of patients on waiting lists. Living donor liver transplantation (LDLT) has flourished over the past decades since its introduction by Raia et al. (1) in 1989.

In the same year when LDLT was reported, sarcopenia was first introduced by Rosenberg (2) to describe agerelated loss of skeletal muscle. It was later defined as the presence of both low muscle mass and function (strength or performance) by the European Working Group on Sarcopenia in Older People (EWGSOP) (3). Although mainly age related, sarcopenia not surprisingly is also seen in patients with liver disease, especially in end-stage liver disease, as the liver is involved in most metabolic process of nutrition absorption and energy extraction (4). In addition, patients with chronic liver disease generally suffer from decreased appetite, anorexia, and malabsorption, which results in insufficient dietary intake and poor nutritional status. In fact, sarcopenia has been reported to present in up to nearly $70 \%$ of LT candidates (5). In terms of the effect on prognosis, sarcopenia has been found to be correlated with unfavorable outcomes in various kinds of malignancies $(6,7)$, and with poor surgical outcomes (8), including LTs (9).

In terms of LDLT, several studies (9-11) have indicated the adverse effects of sarcopenia on recipients' survival. However, the use of sarcopenia as a predictor for posttransplant survival is undoubtedly limited because of the significant heterogeneity among studies. To date, firm diagnostic criteria for sarcopenia have not yet been well established, and calculations of cutoff values for sarcopenia have been based on different statistical methods, including mean value and standard deviations (SDs) $(12,13)$ of healthy controls, receiver operating characteristic (ROC) curves (14), and simple quartile distributions of parameters relating to living donors (15).

In addition, different parameters and measurements have been applied, such as muscle function (hand grip strength) assessment (16) and body composition analysis, including but not limited to bioelectrical impedance analysis (BIA) (17) and computed tomography/magnetic resonance imaging (CT/MRI) scans $(12,18)$. Measurement by radiological imaging is recommended because of its ability to provide precise and direct visualization of body compartments (3). Simple calculated parameters based on transversal and/ or axial psoas muscle thickness $(18,19)$ on CT scan images were used in the earlier period, while normalized (by height) parameters have been preferred in recent years. Normalized parameters mainly include the psoas muscle index (PMI) $(10,12,15)$ and the skeletal muscle index (SMI) $(13,20)$, which are characterized by assessments of systemic or specific skeletal muscle (psoas muscle) mass using certain CT image slides, respectively.

The prognostic effects of sarcopenia in patients undergoing LDLT have been studied in Europe (19-22) and some Asian countries, mainly Japan $(9,13)$ and Korea (11). However, no robust evidence is available for the Chinese population, which has the largest burden of liver cancer, cirrhosis, and related deaths. Furthermore, the number of new cases of cirrhosis and hepatocellular carcinoma (HCC) is expected to continue rising in the coming decade, mainly due to the dramatically increasing prevalence of metabolic disease caused by unhealthy lifestyles and diets $(21,22)$. As a result, a growing number of candidates for LT have been documented in China. Thus, a huge number of LDLTs are required, and the determination of potential risk factors for posttransplant survival are urgently needed in China. In the present study, we aimed to explore the prevalence and effect of sarcopenia defined by PMI value on long-term outcomes among patients undergoing LDLT by using X-tile software (Yale University, New Haven, CT, USA) to determine optimal PMI cutoff values for sarcopenia.

\section{Methods}

\section{Patients and perioperative management}

In total, 117 adult patients underwent LDLT at the Liver Transplantation Center of West China Hospital, Sichuan University, Chengdu, China, between January 2009 and December 2015. We excluded 8 patients who did not undergo preoperative CT scan within a month of preLDLT and 27 patients whose CT scan did not reach the third lumbar vertebra (L3) level. A total of 82 adult LT recipients were included in this study. The study was conducted in accordance with the Declaration of Helsinki (as revised in 2013). The study was approved by the Ethics Committee of West China Hospital, and individual consent for this retrospective analysis was waived.

In all, 365 LDLTs were conducted in our center between 2001 and 2015, and details of surgical procedures and techniques have been reported previously $(23,24)$. A threedimensional reconstruction system, IQQA-liver (EDDA Technology, Princeton, NJ, USA), was used to evaluate hepatic anatomies, including anatomical variations, and 

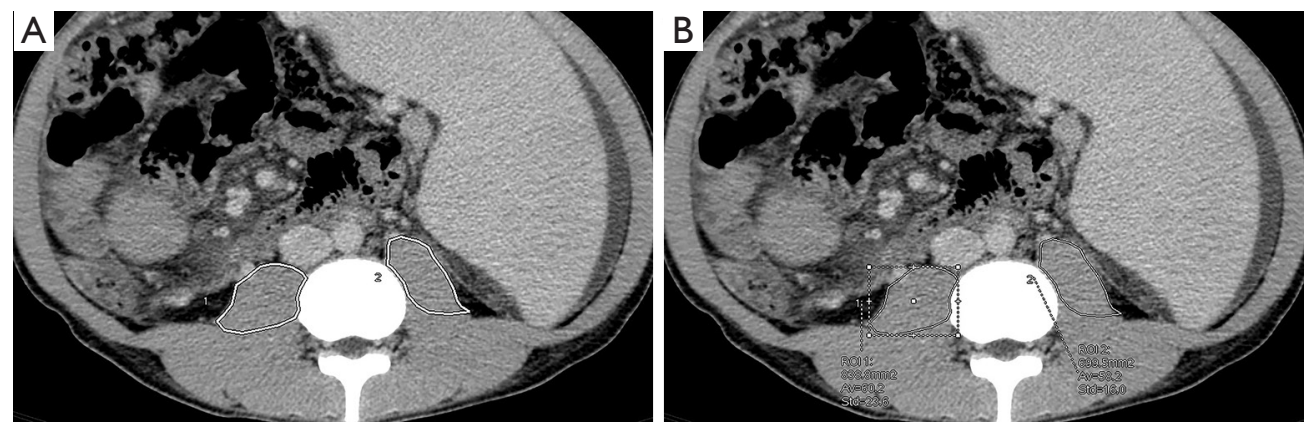

Figure 1 The measurement of psoas muscle area of both sides (1, right; 2, left) on computed tomography slides. (A) An example of the range of bilateral psoas muscle; (B) an example of area measurement of bilateral psoas muscle. The area of the left psoas muscle is $838.8 \mathrm{~mm}^{2}$ and that of the right psoas muscle is $699.5 \mathrm{~mm}^{2}$.

potential liver volumes. Intraoperative parameters were collected, including duration of surgery, blood loss, anhepatic phase, and intraoperative complications. Basic immunosuppression consisted of tacrolimus or cyclosporine and steroids, with or without mycophenolate. The drug concentration of tacrolimus was recorded every other day, while an abdominal ultrasound and routine blood test were performed each day of the first week after transplantation.

\section{Perioperative parameters and long-term outcomes}

Perioperative data of recipients were collected, including age, gender, body mass index (BMI), creatinine, total bilirubin (TBL), albumin, international normalized ratio (INR), Child-Pugh classification, and the model for endstage liver disease score (MELD). Characteristics of donors included donor age, gender, BMI, graft-to-recipient weight ratio (GRWR), and $\mathrm{ABO}$ blood group compatibility. Each recipient was seen weekly for the first month after discharge, and after that monthly for 6 months. Survival events and HCC recurrence with corresponding dates were recorded, and the average follow-up time was $63.3( \pm 39.8)$ months.

\section{Imaging measurement and determination of a cutoff value}

All preoperative CT examinations were performed by using one of two commercially available multidetector CT scanners: Brilliance CT (Philips Healthcare, Best, The Netherlands) or SOMATOM Definition AS+ (Siemens Medical Systems, Erlangen, Germany). A section thickness of $5 \mathrm{~mm}$ with a reconstruction interval of $5 \mathrm{~mm}$, field of view of 330-390 mm, gantry rotation time of 0.5 seconds, tube current-time product of 200-250 mAs, and peak voltage of $120 \mathrm{kVp}$ were used for both CT scanners.
Two experienced abdominal radiologists, who were blinded to the clinical results, independently performed the measurements using the application Reformat on Advantage Workstation Release 4.6 Software (General Electric Company, Milwaukee, Wisconsin, USA). Based on the preoperative CT scan ( $<1$-month pre-LDLT), the 2 radiologists manually measured the area of both sides of the psoas muscles at the third lumbar vertebra (L3) level (Figure 1), independently. PMI was calculated by normalizing the sum of bilateral psoas muscle areas to the square of each patient's height $\left(\mathrm{cm}^{2} / \mathrm{m}^{2}\right)$.

Thereafter, the graphical method of X-tile plots was used to determine the optimal PMI cutoff value. The $\mathrm{X}$-tile was designed to visualize the best cutoff point for outcomes, especially for time-dependent assessments, such as survival outcomes. Based on the cutoff value determined by the $\mathrm{X}$-tile method, patients were divided into 2 groups according to PMI, the sarcopenia and the non-sarcopenia group.

\section{Statistics analysis}

Basic data of recipients and donors are presented as counts (percentages) and means (SDs) where appropriate. An intraclass correlation coefficient (ICC) was calculated to assess interobserver consistency. The sums of bilateral psoas muscle areas from the 2 radiologists were averaged for the subsequent analysis. Based on the PMI and survival data, the $\mathrm{X}$-tile plots showed $\chi^{2} \log$-rank values with a Kaplan-Meier curve and presented the optimal cutoff value for sarcopenia, according to which all patients were divided into 2 groups. The Mann-Whitney $\mathrm{U}$ test and the $\chi^{2}$ test or Fisher's exact test were used for continuous variables and categorical variables, respectively, to compare the basic characteristics of patients with and without sarcopenia. Overall survival 
and disease-free survival were compared using the KaplanMeier method and were assessed with the log-rank test. A Cox hazards regression model was used to explore potential associations between clinical outcomes and various factors. Potential $(\mathrm{P}<0.10)$ and significant $(\mathrm{P}<0.05)$ parameters identified in the univariate analysis were further included in the multivariate regression model. Hazard ratios (HRs) and corresponding $95 \%$ confidence intervals (CIs) were calculated. Statistical analysis was performed using SPSS version 20.0 (IBM Corp, Armonk, NY, USA) and X-tile software v. 3.6.1 (Yale University, New Haven, CT, USA).

\section{Results}

\section{Patient characteristics}

We included 70 males and 12 females in the initial cohort. The X-tile software performed well (cutoff value: $6.25 \mathrm{~cm}^{2} / \mathrm{m}^{2}$ ) when data of both males and females were imported (Figure 2A,2B). However, the definition of sarcopenia is considered to be sex-specific because males have significantly larger psoas muscle area progressive muscular atrophy (PMA) and PMI than do females $(12,25,26)$. However, X-tile was not able to discover a reliable cutoff value based on this small cohort. As a result, the PMI cutoff value for 12 females (Figure $2 C, 2 D$ ) was $2.88 \mathrm{~cm}^{2} / \mathrm{m}^{2}$, which assigned only 1 patient (8.3\%) to the sarcopenia group, which was considerably less than the number assigned in previous reports $(18,26)$. Therefore, we mainly focused on the 70 male patients (Figure $2 E, 2 F$ ) since our data for female patients might not have been sufficiently robust to represent general female candidates for LDLT.

The average age of the 70 male patients was $41.6( \pm 8.9)$ years, while that of the corresponding donors was $38.1( \pm 9.7)$ years. Furthermore, 60 patients $(85.7 \%)$ out of 70 were serum positive for hepatitis B surface antigen (HBsAg), and 67 patients (95.7\%) were pathologically proven to have liver cirrhosis. Etiologic factors for LDLT, as well as other basic characteristics of both recipients and donors are listed in Table S1. The calculated average PMI of recipients was $6.02( \pm 1.60) \mathrm{cm}^{2} / \mathrm{m}^{2}$ as measured by the method described above. The average GRWR was $0.92 \%$ $( \pm 0.20 \%)$, and the average duration of patients' stay in the intensive care unit (ICU) was $11.8( \pm 7.2)$ days.

\section{Interobserver consistency}

The interobserver agreement between the 2 radiologists was very good for areas of both the right (ICC $=0.924 ; 95 \%$ CI, $0.885-0.950)$ and left (ICC $=0.901 ; 95 \%$ CI, 0.851-0.935) psoas muscle.

\section{Cutoff value for PMI and baseline comparison}

For males, the PMI cutoff point was $6.25 \mathrm{~cm}^{2} / \mathrm{m}^{2}$, according to which 38 patients $(54.3 \%)$ were diagnosed with sarcopenia. Table 1 shows the comparison of clinicopathological characteristics between patients with and without sarcopenia. Patients with sarcopenia presented with lower BMI $(\mathrm{P}<0.001)$ and albumin $(\mathrm{P}=0.029)$, along with higher levels of TBL $(\mathrm{P}=0.005)$ and higher MELD scores $(\mathrm{P}=0.004)$. Patients with sarcopenia tended to have more pretransplant complications, but most comparisons were not significantly different $(\mathrm{P}>0.05)$, except for progressive hyperbilirubinemia $(\mathrm{P}=0.031)$. Otherwise, the 2 groups of recipients had similar $(\mathrm{P}>0.05)$ ages at transplantation, pretransplant creatinine, proportion of pretransplant hospital stay, and etiology of liver diseases. In regard to donor characteristics and surgical procedures, no significant differences were revealed between the 2 groups, including in donor gender, age, GRWR, ABO-compatible rates, operation, or cold ischemia times.

\section{Intra- and postoperative complications}

Two patients experienced intraoperative complications, including one cardiac arrest in the sarcopenia group and one massive hemorrhage in the non-sarcopenia group.

Table 2 shows the intraoperative and postoperative complications of both groups; the latter includes early- and late-phase complications. No statistical differences $(\mathrm{P}>0.05)$ were detected between the 2 groups in terms of either earlyor late-phase complications.

\section{Overall survival after LDLT}

All patients were followed up until July 1, 2019, or until death (from any reason). After an average of $63.3 \pm 39.4$ months of follow-up, 21 patients died after LDLT, 16 in the sarcopenia group and 5 in the non-sarcopenia group. The causes of death for patients with sarcopenia were HCC recurrence $(\mathrm{N}=6)$, graft failure $(\mathrm{N}=4)$, pulmonary infection $(\mathrm{N}=2)$, and others $(\mathrm{N}=4)$; meanwhile, 5 patients without sarcopenia died from HCC recurrence $(\mathrm{N}=2)$, graft failure $(\mathrm{N}=2)$, and other causes $(\mathrm{N}=1)$. Patients with sarcopenia had significantly reduced cumulative overall survival rates $(\mathrm{P}<0.05)$ than did 

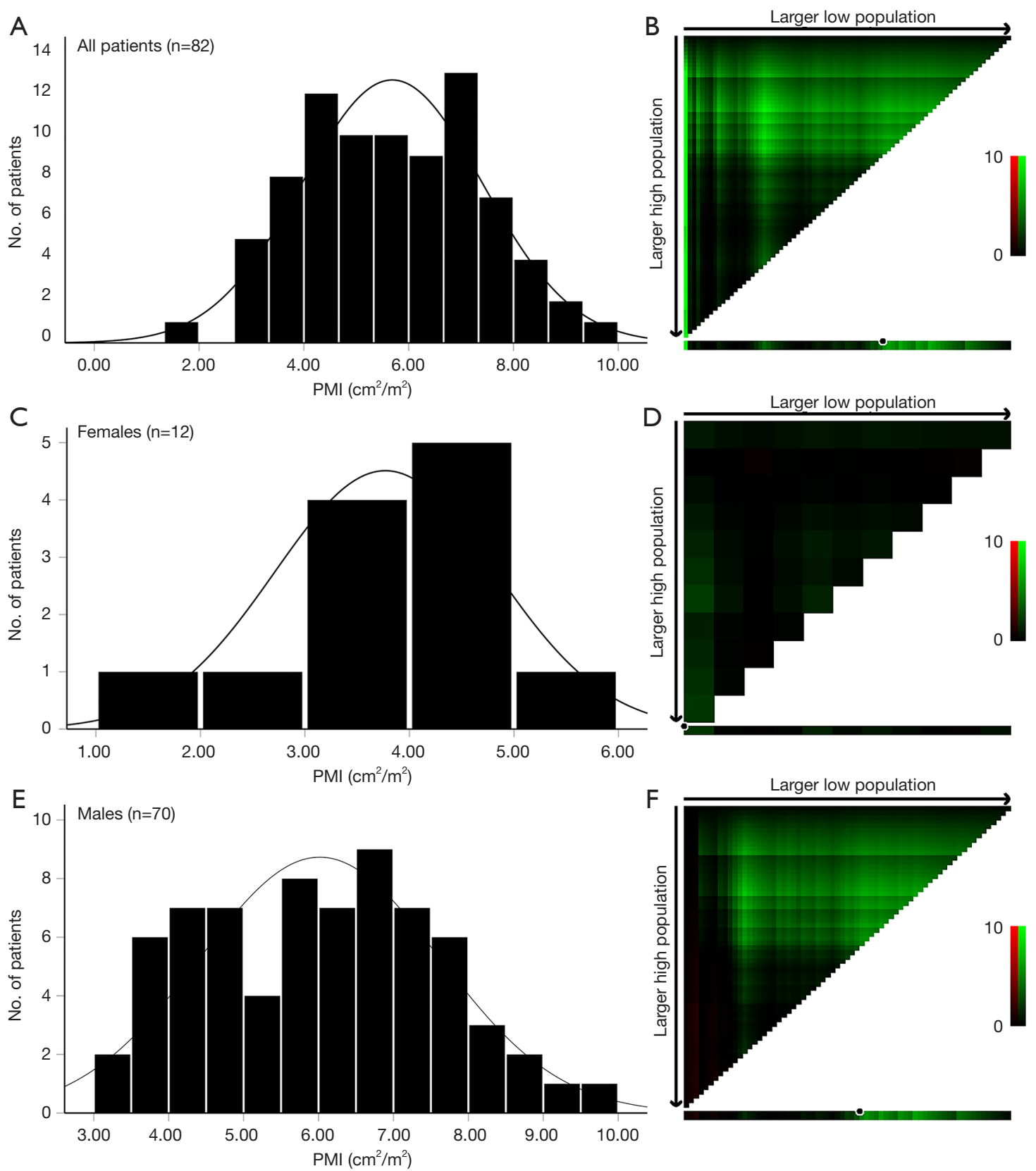

Figure 2 Distributions of psoas muscle index and calculation of optimal cutoff values. Histograms and X-tile plots of psoas muscle index according to survival outcomes of all patients (A,B), females (C,D), and males (E,F). PMI, psoas muscle index.

those without sarcopenia (Figure 3). The 1-, 3-, and 5-year survival rates were $71.1 \%, 65.8 \%$, and $63.2 \%$, respectively, for the sarcopenia group; and $96.9 \%, 87.5 \%$, and $83.7 \%$, respectively, for the non-sarcopenia group.

In univariate analysis (Table 3), decreased overall survival was related to $(\mathrm{P}<0.05)$ sarcopenia and pretransplant hospital stay, while it was not significantly associated with age, BMI, portal vein thrombosis (PVT), ChildPugh C class, or a MELD score $>20$. In multivariate analysis, sarcopenia and pretransplant hospital stay were identified as being significantly associated $(\mathrm{P}<0.05)$ with worse posttransplant overall survival, resulting in HRs of 3.22 (95\% CI, 1.15-8.98) and 2.46 (95\% CI, 1.00-6.05), respectively, while recipients' age (95\% CI, 0.99-1.10) 
Table 1 Baseline characteristics of patients with and without sarcopenia

\begin{tabular}{|c|c|c|c|}
\hline Characteristics & Sarcopenia $(n=38)$ & Non-sarcopenia $(n=32)$ & $P$ \\
\hline Age, years & $43( \pm 9.7)$ & $40( \pm 7.7)$ & 0.157 \\
\hline $\mathrm{BMI}, \mathrm{kg} / \mathrm{m}^{2}$ & $21.6( \pm 2.2)$ & $24.5( \pm 2.8)$ & $<0.001$ \\
\hline Creatinine, $\mathrm{mmol} / \mathrm{L}$ & $74.6( \pm 21.8)$ & $74.1( \pm 13.9)$ & 0.908 \\
\hline TBL, mmol/L & $88.9( \pm 11.9)$ & $27.6( \pm 18.9)$ & 0.005 \\
\hline INR & $1.48( \pm 0.47)$ & $1.27( \pm 0.27)$ & 0.033 \\
\hline HBsAg, n (\%) & $30(78.9)$ & $30(93.8)$ & 0.097 \\
\hline AFP >400 ng/mL, n (\%) & $9(23.7)$ & $9(28.1)$ & 0.786 \\
\hline Refractory ascites, n (\%) & $9(23.7)$ & $2(6.3)$ & 0.055 \\
\hline History of varicose veins bleeding, $\mathrm{n}(\%)$ & $2(5.3)$ & $1(3.1)$ & 1.000 \\
\hline Spontaneous bacterial peritonitis, $\mathrm{n}(\%)$ & $2(5.3)$ & $0(0)$ & 0.496 \\
\hline Hepatic encephalopathy, n (\%) & $3(7.9)$ & $0(0)$ & 0.245 \\
\hline Pretransplant infection, n (\%) & $4(10.5)$ & $1(3.1)$ & 0.366 \\
\hline Pretransplant hospital stay, n (\%) & $17(44.7)$ & $13(40.6)$ & 0.811 \\
\hline \multicolumn{4}{|l|}{ Donor and surgical factors } \\
\hline Donor age, years & $38.4( \pm 10.2)$ & $37.7( \pm 9.3)$ & 0.747 \\
\hline Cold ischemia time, hour & $2.1( \pm 2.2)$ & $2.2( \pm 2.7)$ & 0.818 \\
\hline
\end{tabular}

BMI, body mass index; TBL, total bilirubin; INR, international normalized ratio; HBsAg, hepatitis B surface antigen; AFP, alpha fetoprotein; MELD, model for end-stage liver disease score; GRWR, graft-to-recipient weight ratio.

and the presence of PVT (95\% CI, 0.84-64.3) were not independent predictors.

\section{Overall survival and disease-free survival in patients with HCC after LDLT}

There were 50 recipients $(71.4 \%)$ with HCC, and 24 (48.0\%) of these were categorized as having sarcopenia according to the cutoff value. Patients with sarcopenia had worse overall survival $(\mathrm{P}=0.052)$ than did those without
(Figure 4A). The 1-, 3-, and 5-year cumulative survival rates were $92.3 \%, 84.6 \%$, and $78.1 \%$, respectively, in the non-sarcopenia group; and $75.0 \%, 62.5 \%$, and $58.3 \%$, respectively, in the sarcopenia group. Patients with HCC meeting the Milan criteria had better cumulative survival $(\mathrm{P}=0.025)$ when compared to those patients not meeting the Milan criteria (Figure 4B), with 1-, 3-, and 5-year survival rates of $91.7 \%, 87.5 \%, 87.5 \%$; and $80.8 \%, 65.4 \%, 57.7 \%$, respectively.

In univariate analysis (Table 4), HCC meeting Milan 
Table 2 Intraoperative and postoperative complications

\begin{tabular}{|c|c|c|c|}
\hline Complication category & Sarcopenia (N=38), n (\%) & Non-sarcopenia (N=32), n (\%) & $\mathrm{P}$ \\
\hline Early postoperative complications & $14(36.8)$ & $16(50.0)$ & 1.000 \\
\hline Biliary stenosis & $0(0)$ & $1(3.1)$ & 1.000 \\
\hline Biliary leakage & $1(2.6)$ & $0(0)$ & 0.457 \\
\hline Intraabdominal collection & $7(18.4)$ & 7 (21.9) & 0.771 \\
\hline Postoperative bleeding & $0(0)$ & $3(9.4)$ & 0.245 \\
\hline Portal vein thrombosis & $1(2.6)$ & $1(3.1)$ & 1.000 \\
\hline Portal vein Stenosis & $2(5.3)$ & $0(0)$ & 0.205 \\
\hline Late postoperative complications & $8(21.1)$ & $5(15.6)$ & 0.232 \\
\hline Arterial thrombosis & $1(2.6)$ & $0(0)$ & 0.457 \\
\hline Portal vein thrombosis & $0(0)$ & $2(6.3)$ & 0.496 \\
\hline Biliary leakage & $1(2.6)$ & $3(9.4)$ & 0.620 \\
\hline Biliary anastomosis stenosis & $2(5.3)$ & $1(3.1)$ & 0.589 \\
\hline
\end{tabular}

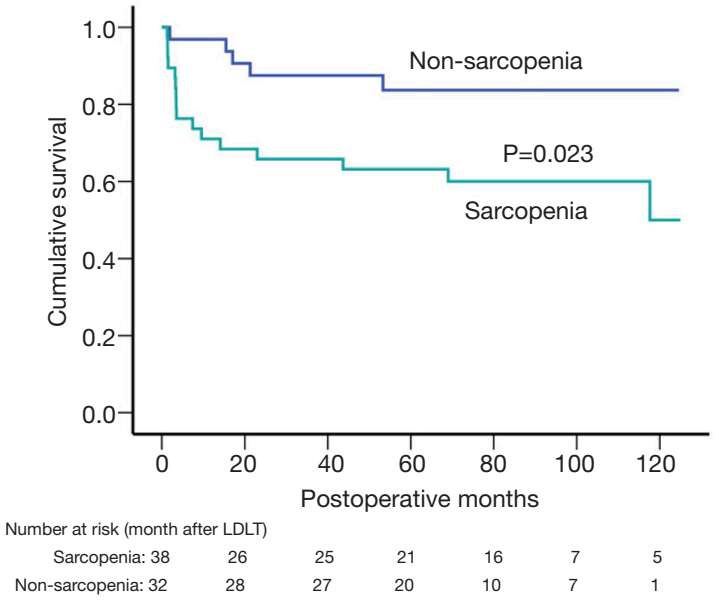

Figure 3 Cumulative overall survival in patients with and without sarcopenia.

criteria was associated with significantly reduced mortality $(\mathrm{P}=0.035)$, while sarcopenia was related to an insignificantly increased risk of mortality $(\mathrm{P}=0.062)$. Other recipient and donor factors affecting posttransplant survival $(\mathrm{P}>0.05)$ were not identified. In multivariate analysis, meeting Milan criteria remained an independent predictive factor of overall survival, resulting in a HR of 0.31 (95\% CI, 0.10-1.00), while sarcopenia exhibited a nonsignificant increased HR $(\mathrm{P}=0.095)$.

In regard to recurrence-free survival (RFS), patients in the sarcopenia group had a decreased cumulative RFS $(\mathrm{P}=0.030)$ compared to those in the non-sarcopenia group (Figure 4C), with a 1-, 3-, and 5-year RFS of 75.4\%, 55.3\%, $44.0 \%$; and $92.05 \%, 80.0 \%$, and $73.3 \%$, respectively. Sarcopenia was associated with a 2.87 -fold increased risk of recurrence (95\% CI, 1.06-7.80) in univariate analysis, but was not an independent risk factor for RFS $(\mathrm{P}=0.062)$. In multivariate analysis. On the other hand, meeting Milan criteria was observed to have a protective effect on RFS, resulting in a $65.7 \%$ reduced recurrence risk $(95 \% \mathrm{CI}$, 0.12-0.98) and an improved cumulative RFS (Figure 4D). Other recipient and donor factors were not significantly related to RFS $(\mathrm{P}>0.05)$, with details shown in Table 5 .

\section{Discussion}

This retrospective study first indicated a significant adverse effect of sarcopenia defined by PMI on long-term survival 
Table 3 Univariate and multivariate analysis of prognostic factors for overall survival in 70 male patients after living donor liver transplantation

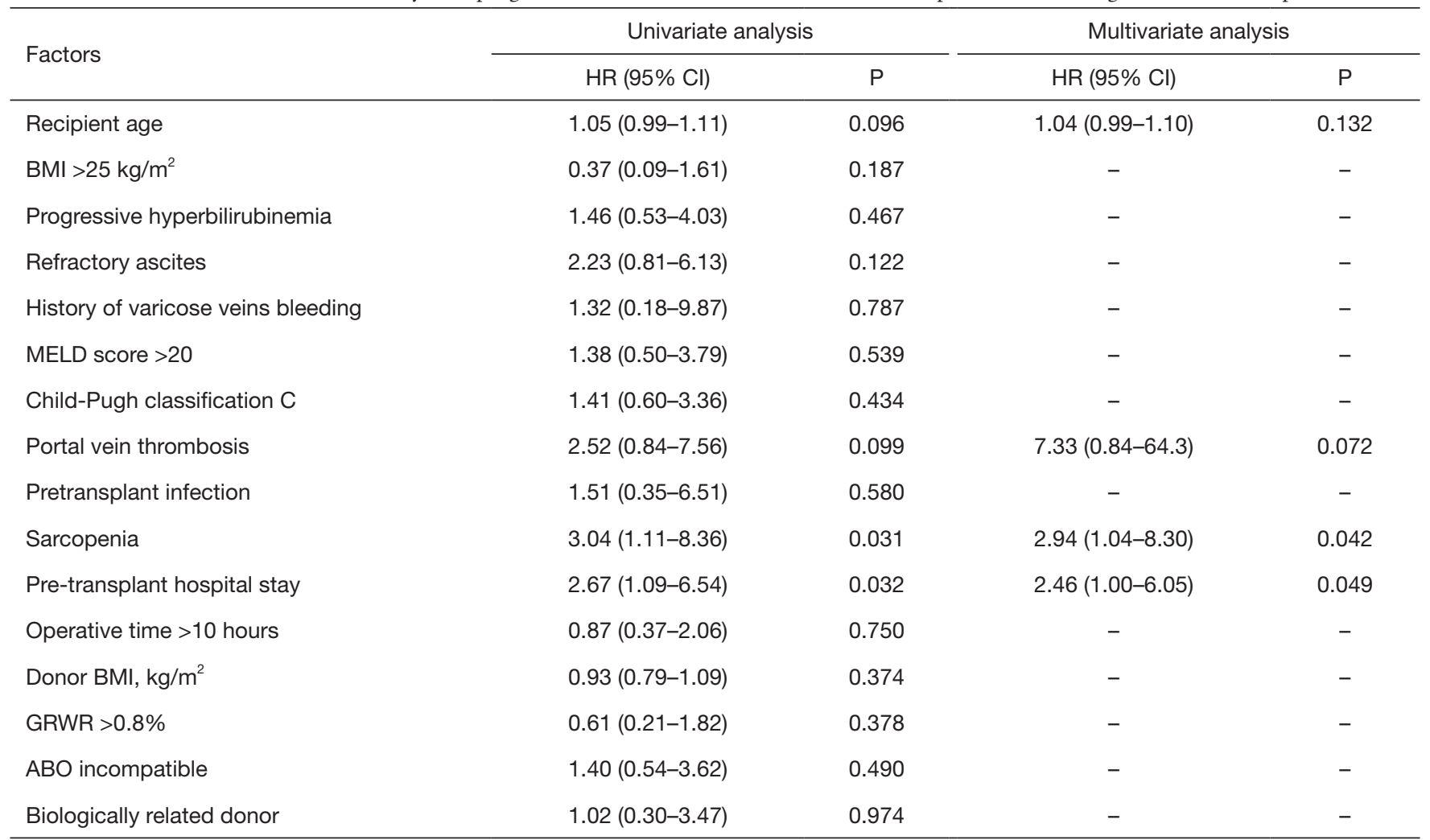

HR, hazard ratio; Cl, confidence interval; BMI, body mass index; MELD, model for end-stage liver disease score; GRWR, graft-to-recipient weight ratio; HR, hazard ratio.

after LDLT among recipients in China, where a large number of LDLTs are required each year.

Sarcopenia, a reliable reflection of malnutrition, is highly prevalent in patients with chronic digestive system diseases and end-stage liver disease in particular (20), and 38 recipients (54.3\%) were diagnosed with sarcopenia in the current study according to our definition. Unsurprisingly, the importance of sarcopenia has been of great clinical interest over the past decade, as it has been found to be related to worse outcomes in various types of malignancies $(6,7)$ and renal transplantation (27). Previous studies revealed sarcopenia as a negative prognostic factor for survival after LDLT, but parameters to define sarcopenia and determination of cutoff points vary between studies, which significantly limits its application.

Various methods have been used to evaluate body composition, the major parameter for assessing sarcopenia. Kaido et al. (9) identified sarcopenia using multifrequency BIA and demonstrated its significant association with worse overall survival after LDLT. However, BIA examination is not included in the routine management of patients undergoing LDLT on admission, at least not in most transplant centers in China, and some patients cannot complete BIA examination due to their poor general condition. As a result, they excluded 68 patients (35.4\%) for not completing BIA examination (9). As the EWGSOP has defined CT and MRI as gold standards for the assessment of body muscle mass (3), radiological imaging techniques are applied in most studies (28), especially CT, while dual energy X-ray absorptiometry (DXA) is recommended as a preferred alternative method.

Based on radiological techniques, core muscles (29) are well established predictors for sarcopenia, as they can better characterize the overall health condition than can BMI and BIA, especially in patients with obesity or ascites, for whom application of the latter two methods is significantly limited. Parameters based on the psoas muscle are frequently selected to evaluate sarcopenia, for instance, PMI or PMA (psoas muscle area), because the psoas muscle is the major component of core muscles, and it is feasible to precisely 

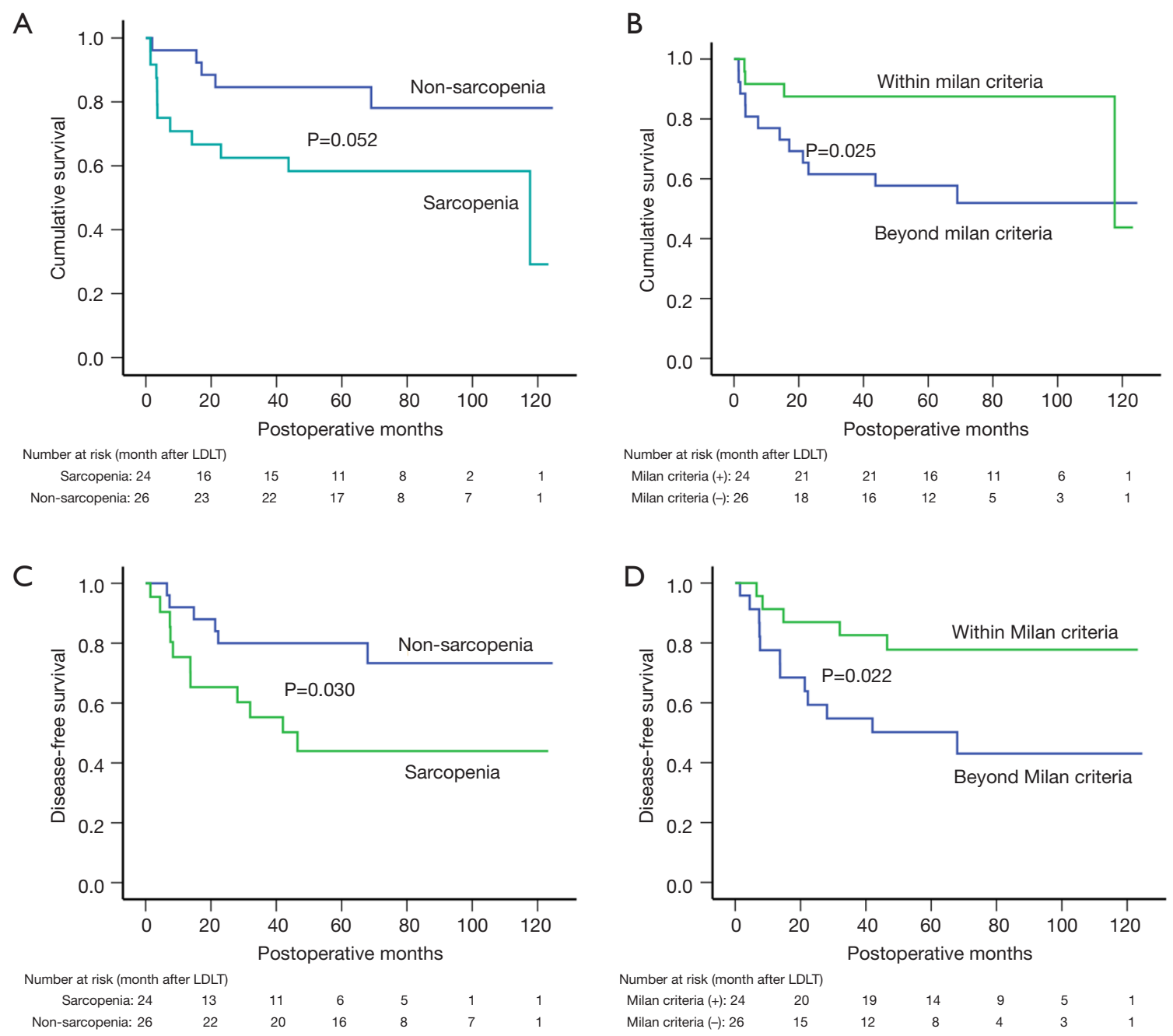

Figure 4 Cumulative overall survival and recurrence-free survival after living donor liver transplantation stratified by sarcopenia and meeting Milan criteria. (A,B) Overall survival according to sarcopenia and Milan criteria status. (C,D) Recurrence-free survival according to sarcopenia and Milan criteria status.

measure it on cross-sectional imaging. In the present study, we found a significant association between sarcopenia defined by PMI and poor overall survival after LDLT, which is line with a previous study (26). However, Ebadi et al. (20) stated that PMI was not a promising predictor of waitlist mortality in cirrhotic patients. Nonetheless, the risk of posttransplant mortality is supposed to differ from that of waitlist mortality, as LDLT simultaneously replaces the diseased liver with a new one and improves the functioning of related organs; thus, the potential for PMI as a predictor of posttransplant survival should not be dismissed. In fact, several studies $(14,15,25)$ recommend the PMI as a useful predictor for survival after LDLT. In addition, Golse et al. (26) compared the 4 most popular methods reported in the literature and concluded that preoperative PMI and PMA had superior performances compared to SMI and PMA. Last but not the least, the psoas muscle is easily recognized on a CT slice, and it takes only a few seconds to complete the measurements, which facilitates its clinical application.

Unfortunately, the mechanism of muscle loss and its adverse effect on postoperative outcomes is not fully understood. Muscle mass is a key indicator of proteinenergy metabolism, and protein-energy malnutrition has been identified as a risk factor of increased postoperative mortality (9). Muscles provide most blood amino acids, 
Table 4 Univariate and multivariate analysis of prognostic factors for overall survival in 50 HCC patients after living donor liver transplantation

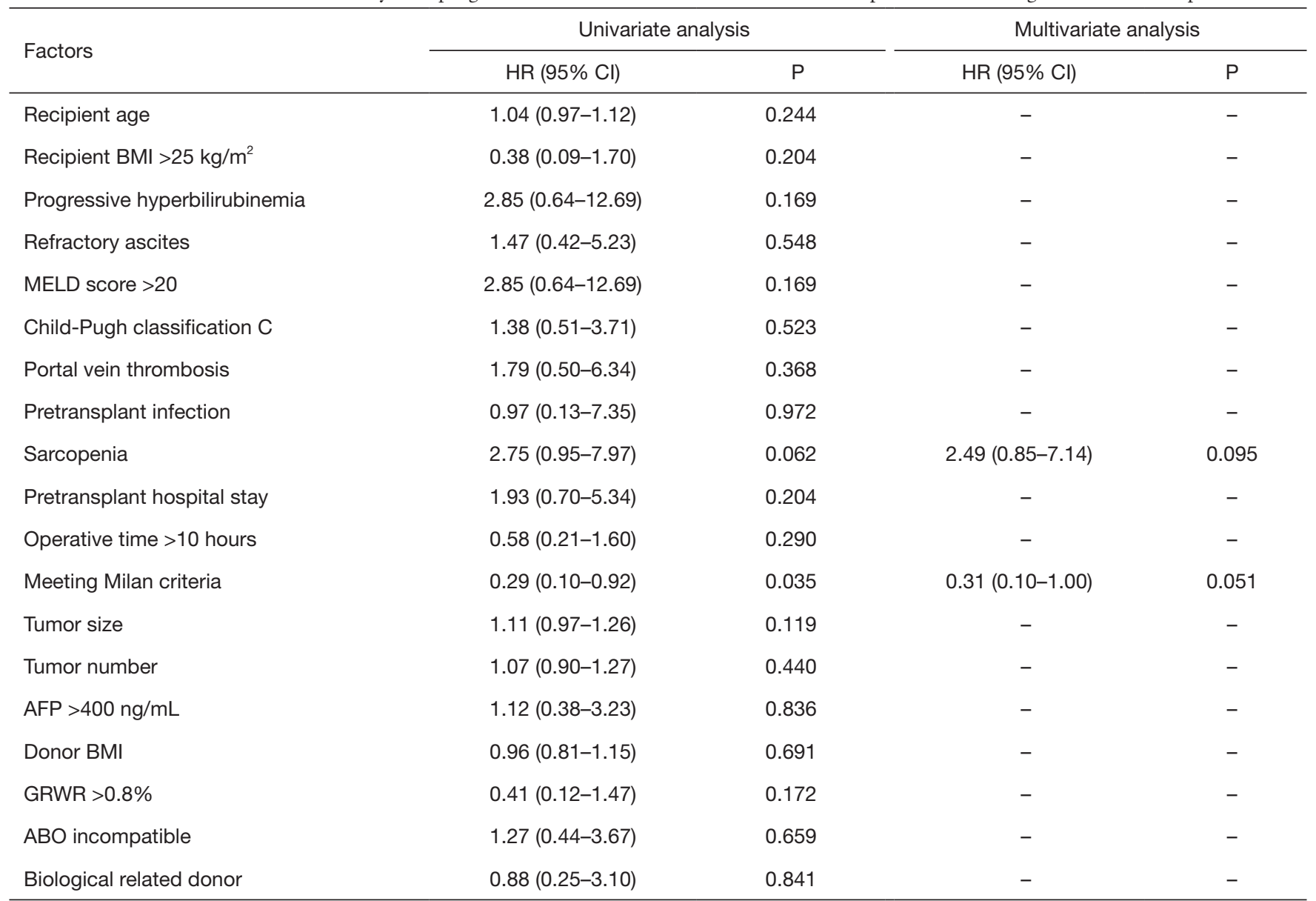

HCC, hepatocellular carcinoma; HR, hazard ratio; Cl, confidence interval; BMI, body mass index; MELD, model for end-stage liver disease score; AFP, alpha fetoprotein; GRWR, graft-to-recipient weight ratio; HR, hazard ratio.

which are necessary for the synthesis of new proteins for other tissues and organs, including those organs that are essential for survival, such as the heart, liver and brain, especially in the postabsorptive state when there is very little surplus protein available (30). Consequently, sufficient muscle mass is the principal guarantee of constant protein mass in essential tissues and organs that are extremely important for survival. Furthermore, amino acids derived from the breakdown of muscle also serve as the major reservoir for the physiologic response in the stress state, including in cancer, infection, and traumatic injury (31). In the current study, $71.4 \%$ of patients presented with liver cancer, 13 patients died of cancer recurrence and progression, and 8 deaths were due to sepsis. In the setting of infection or cancer, the recovery process requires more amino acids to accelerate the synthesis of acute phase protein and protein involved in immune response. It is exceedingly difficult to reverse the net breakdown of muscle protein even with aggressive nutrition support, as the stressed state imposes greater requirements of amino acids than does fasting (31). Moreover, blood amino acids derived from protein breakdown of muscle are also the precursors for hepatic gluconeogenesis in which synthesis is enhanced in conditions of stress $(32,33)$. Hence, we believe the loss of muscle mass is a potential explanation for the significantly unfavorable survival in the sarcopenia group. On the other hand, patients who were hospitalized before LDLT experienced worse posttransplant survival than those who were not. These patients were supposed to have a poor general condition at transplant, complications demanding medical interference, or diseases in organs other than the liver that accounted for the poor recovery after 
Table 5 Univariate and multivariate analysis of prognostic factors for disease-free survival in 50 HCC patients after living donor liver transplantation

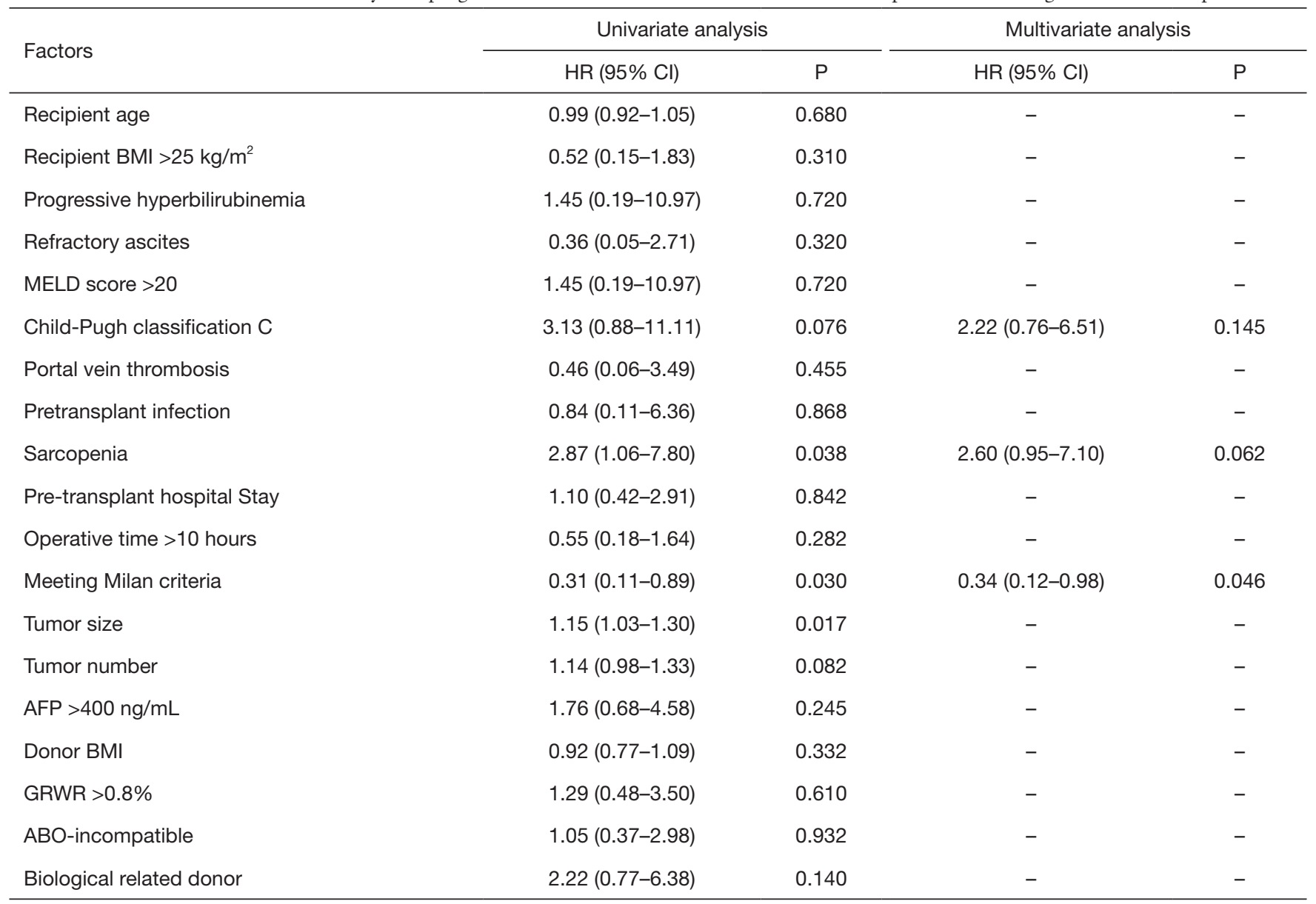

HCC, hepatocellular carcinoma; HR, hazard ratio; Cl, confidence interval; BMI, body mass index; MELD, model for end-stage liver disease score; AFP, alpha fetoprotein; GRWR, graft-to-recipient weight ratio; HR, hazard ratio.

transplantation.

Previous studies $(26,34)$ have indicated that sarcopenia is related to an up to 17.6 -fold increased mortality after LDLT among cirrhotic recipients; however, little evidence is available for candidates with HCC. Kim et al. (11) reported that no significant difference of overall survival was detected between patients with and without sarcopenia after LDLT; however, all patients included had advanced HCC not meeting Milan criteria. In the present study, similar results were found in the $50 \mathrm{HCC}$ cases, of which about half met Milan criteria. It appears that sarcopenia defined by $\mathrm{PMI}$ is not an independent predictor for posttransplant survival in recipients with $\mathrm{HCC}(\mathrm{P}=0.095)$. The prevalence and severity of sarcopenia are greater in decompensated cirrhotic candidates than in those with HCC; thus, the influence of sarcopenia on recipients' survival is possibly overestimated in patients with HCC when they are analyzed together with cirrhotic patients.

In terms of HCC recurrence, the effect of muscle loss is controversial; however, little research has been conducted regarding this issue. In a Korean investigation (11), sarcopenia defined by height-normalized psoas muscle thickness was reported to be associated with an over 9-fold increased recurrence risk after LDLT in patients with HCC not meeting Milan criteria. In another single study conducted in Europe (35) and involving deceased donor LT, sarcopenia defined by skeletal muscle indices did not worsen HCC recurrence. In our study, $48.0 \%$ of HCC cases met Milan criteria, and they presented with higher PMI $\left(6.61 \pm 1.74\right.$ vs. $\left.5.64 \pm 1.44 \mathrm{~cm}^{2} / \mathrm{m}^{2}\right)$ than did those exceeding Milan criteria, possibly because tumor progression accelerated skeletal muscle loss and led to cachexia. It is 
conceivable that sarcopenia and its corresponding metabolic disorder promote carcinogenesis, although sarcopenia is not an independent poor predictor of RFS after LDLT in multivariate analysis $(\mathrm{P}=0.062)$. The limited number of HCC cases and the interaction between sarcopenia and meeting Milan criteria may account for the nonsignificant result. One possible mechanism for this is related to the role that skeletal muscle plays in the inflammation and immunoregulation involved in carcinogenesis. As the largest secretary organ, skeletal muscle produces and releases cytokines and other functional peptides, as known as myokines, which are capable of counteracting the negative influence of proinflammatory adipokines (36) and of mediating immunoregulatory effects. Skeletal muscle is thought to be a robust mediator of circulating interleukin 6 (IL-6), which has been suggested as being involved in the anti-inflammatory process and carcinogenesis of HCC, especially in obese individuals (37). Furthermore, myokines improve insulin sensitivity through activating AMP-activated protein kinase (AMPK) or directly affecting insulin secretion by pancreatic cell (38); thus, muscle loss may result in insulin resistance, which is related to various chronic diseases and carcinogenesis $(11,39)$. Given the uncertain result based on limited HCC cases in the current study, prospectively designed research with larger sample sizes specifically focused on HCC is urgently needed to confirm the effect of sarcopenia on the risk of tumor recurrence after LDLT and its pathogenesis, given posttransplant recurrence is the predominate causation of recipients' death.

The current study has several limitations. First, we only analyzed male patients because of the very limited number and the poor performance of cutoff calculation in females. Hepatitis B virus (HBV)-related cirrhosis and/or HCC is the major cause of being a LT candidate in China, and males are far more vulnerable than are females. For instance, in our original cohort, $96(82.1 \%)$ out of 117 were males, 60 (85.7\%) of whom were HBV-infected male patients included in the analysis. In addition, sarcopenia is more prevalent in men than in women $(20,34)$, and thus the focus of the current study is justified. Second, the limited number of HCC cases possibly accounts for the uncertain association between sarcopenia and HCC recurrence after LDLT. Third, given its retrospective nature, our findings from a single center need to be validated in a prospective study.

In conclusion, the definition of sarcopenia based on PMI value and dynamic time-dependent outcomes of recipients is feasible and reliable. Sarcopenia according to a PMI cutoff value is an independent predictor of poor overall survival after LDLT, at least in China. However, sarcopenia is not independently related to posttransplant HCC recurrence although a tendency towards significance was observed. Further prospective studies with larger sample sizes are needed to confirm the effect of sarcopenia on the risk of HCC recurrence after LDLT.

\section{Acknowledgments}

Funding: This study was supported by grants from the 1.3.5 Project for the Discipline of Excellence, West China Hospital, Sichuan University (No. ZY2017308). Data were derived from the records of the Chinese Liver Transplant Registry.

\section{Footnote}

Conflicts of Interest: All authors have completed the ICMJE uniform disclosure form (available at https://dx.doi. org/10.21037/qims-21-314). The authors have no conflicts of interest to declare.

Ethical Statement: The authors are accountable for all aspects of the work in ensuring that questions related to the accuracy or integrity of any part of the work are appropriately investigated and resolved. The study was conducted in accordance with the Declaration of Helsinki (as revised in 2013). The study was approved by the Ethics Committee of West China Hospital, and individual consent for this retrospective analysis was waived.

Open Access Statement: This is an Open Access article distributed in accordance with the Creative Commons Attribution-NonCommercial-NoDerivs 4.0 International License (CC BY-NC-ND 4.0), which permits the noncommercial replication and distribution of the article with the strict proviso that no changes or edits are made and the original work is properly cited (including links to both the formal publication through the relevant DOI and the license). See: https://creativecommons.org/licenses/by-nc-nd/4.0/.

\section{References}

1. Raia S, Nery JR, Mies S. Liver transplantation from live donors. Lancet 1989;2:497.

2. Rosenberg I. Epidemiologic and methodologic problems 
in determining nutritional status of older persons. (Summary comments). Am J Clin Nutr 1989;50:1231-3.

3. Cruz-Jentoft AJ, Baeyens JP, Bauer JM, Boirie Y, Cederholm T, Landi F, Martin FC, Michel JP, Rolland Y, Schneider SM, Topinková E, Vandewoude M, Zamboni M; European Working Group on Sarcopenia in Older People. Sarcopenia: European consensus on definition and diagnosis: Report of the European Working Group on Sarcopenia in Older People. Age Ageing 2010;39:412-23.

4. Anand AC. Nutrition and Muscle in Cirrhosis. J Clin Exp Hepatol 2017;7:340-57.

5. Cruz RJ Jr, Dew MA, Myaskovsky L, Goodpaster B, Fox K, Fontes P, DiMartini A. Objective radiologic assessment of body composition in patients with end-stage liver disease: going beyond the BMI. Transplantation 2013;95:617-22.

6. Feliciano EMC, Kroenke CH, Meyerhardt JA, Prado CM, Bradshaw PT, Kwan ML, Xiao J, Alexeeff S, Corley D, Weltzien E, Castillo AL, Caan BJ. Association of Systemic Inflammation and Sarcopenia With Survival in Nonmetastatic Colorectal Cancer: Results From the C SCANS Study. JAMA Oncol 2017;3:e172319.

7. Caan BJ, Cespedes Feliciano EM, Prado CM, Alexeeff S, Kroenke CH, Bradshaw P, Quesenberry CP, Weltzien EK, Castillo AL, Olobatuyi TA, Chen WY. Association of Muscle and Adiposity Measured by Computed Tomography With Survival in Patients With Nonmetastatic Breast Cancer. JAMA Oncol 2018;4:798-804.

8. Mueller N, Murthy S, Tainter CR, Lee J, Riddell K, Fintelmann FJ, Grabitz SD, Timm FP, Levi B, Kurth T, Eikermann M. Can Sarcopenia Quantified by Ultrasound of the Rectus Femoris Muscle Predict Adverse Outcome of Surgical Intensive Care Unit Patients as well as Frailty? A Prospective, Observational Cohort Study. Ann Surg 2016;264:1116-24.

9. Kaido T, Ogawa K, Fujimoto Y, Ogura Y, Hata K, Ito T, Tomiyama K, Yagi S, Mori A, Uemoto S. Impact of sarcopenia on survival in patients undergoing living donor liver transplantation. Am J Transplant 2013;13:1549-56.

10. Hamaguchi Y, Kaido T, Okumura S, Fujimoto Y, Ogawa K, Mori A, Hammad A, Tamai Y, Inagaki N, Uemoto S. Impact of quality as well as quantity of skeletal muscle on outcomes after liver transplantation. Liver Transpl 2014;20:1413-9.

11. Kim YR, Park S, Han S, Ahn JH, Kim S, Sinn DH, Jeong WK, Ko JS, Gwak MS, Kim GS. Sarcopenia as a predictor of post-transplant tumor recurrence after living donor liver transplantation for hepatocellular carcinoma beyond the Milan criteria. Sci Rep 2018;8:7157.
12. Hamaguchi Y, Kaido T, Okumura S, Kobayashi A, Hammad A, Tamai Y, Inagaki N, Uemoto S. Proposal for new diagnostic criteria for low skeletal muscle mass based on computed tomography imaging in Asian adults. Nutrition 2016;32:1200-5.

13. Hamaguchi $Y$, Kaido T, Okumura S, Kobayashi A, Shirai H, Yagi S, Kamo N, Okajima H, Uemoto S. Impact of Skeletal Muscle Mass Index, Intramuscular Adipose Tissue Content, and Visceral to Subcutaneous Adipose Tissue Area Ratio on Early Mortality of Living Donor Liver Transplantation. Transplantation 2017;101:565-74.

14. Hamaguchi Y, Kaido T, Okumura S, Kobayashi A, Shirai H, Yagi S, Hammad A, Okajima H, Uemoto S. Proposal of Muscle-MELD Score, Including Muscularity, for Prediction of Mortality After Living Donor Liver Transplantation. Transplantation 2016;100:2416-23.

15. Izumi $T$, Watanabe J, Tohyama $T$, Takada Y. Impact of psoas muscle index on short-term outcome after living donor liver transplantation. Turk J Gastroenterol 2016;27:382-8.

16. Harimoto N, Yoshizumi T, Izumi T, Motomura T, Harada N, Itoh S, Ikegami T, Uchiyama H, Soejima Y, Nishie A, Kamishima T, Kusaba R, Shirabe K, Maehara Y. Clinical Outcomes of Living Liver Transplantation According to the Presence of Sarcopenia as Defined by Skeletal Muscle Mass, Hand Grip, and Gait Speed. Transplant Proc 2017;49:2144-52.

17. Kaido T, Tamai Y, Hamaguchi Y, Okumura S, Kobayashi A, Shirai H, Yagi S, Kamo N, Hammad A, Inagaki N, Uemoto S. Effects of pretransplant sarcopenia and sequential changes in sarcopenic parameters after living donor liver transplantation. Nutrition 2017;33:195-8.

18. Masuda T, Shirabe K, Ikegami T, Harimoto N, Yoshizumi T, Soejima Y, Uchiyama H, Ikeda T, Baba H, Maehara Y. Sarcopenia is a prognostic factor in living donor liver transplantation. Liver Transpl 2014;20:401-7.

19. Durand F, Buyse S, Francoz C, Laouénan C, Bruno O, Belghiti J, Moreau R, Vilgrain V, Valla D. Prognostic value of muscle atrophy in cirrhosis using psoas muscle thickness on computed tomography. J Hepatol 2014;60:1151-7.

20. Ebadi M, Wang CW, Lai JC, Dasarathy S, Kappus MR, Dunn MA, Carey EJ, Montano-Loza AJ; From the Fitness, Life Enhancement, and Exercise in Liver Transplantation (FLEXIT) Consortium. Poor performance of psoas muscle index for identification of patients with higher waitlist mortality risk in cirrhosis. J Cachexia Sarcopenia Muscle 2018;9:1053-62.

21. Wu J, Yang S, Xu K, Ding C, Zhou Y, Fu X, Li Y, Deng 
M, Wang C, Liu X, Li L. Patterns and Trends of Liver Cancer Incidence Rates in Eastern and Southeastern Asian Countries (1983-2007) and Predictions to 2030. Gastroenterology 2018;154:1719-1728.e5.

22. Tan $Y$, Zhang X, Zhang W, Tang L, Yang H, Yan K, Jiang L, Yang J, Li C, Yang J, Wen T, Tang H, Yan L. The Influence of Metabolic Syndrome on the Risk of Hepatocellular Carcinoma in Patients with Chronic Hepatitis B Infection in Mainland China. Cancer Epidemiol Biomarkers Prev 2019;28:2038-46.

23. Zhang X, Yang J, Yan L, Li B, Wen T, Xu M, Wang W, Zhao J, Wei Y. Comparison of laparoscopy-assisted and open donor right hepatectomy: a prospective case-matched study from china. J Gastrointest Surg 2014;18:744-50.

24. Song JL, Yang J, Wu H, Yan LN, Wen TF, Wei YG, Yang JY. Pure laparoscopic right hepatectomy of living donor is feasible and safe: a preliminary comparative study in China. Surg Endosc 2018;32:4614-23.

25. Kalafateli M, Mantzoukis K, Choi Yau Y, Mohammad AO, Arora S, Rodrigues S, de Vos M, Papadimitriou K, Thorburn D, O'Beirne J, Patch D, Pinzani M, Morgan MY, Agarwal B, Yu D, Burroughs AK, Tsochatzis EA. Malnutrition and sarcopenia predict post-liver transplantation outcomes independently of the Model for End-stage Liver Disease score. J Cachexia Sarcopenia Muscle 2017;8:113-21.

26. Golse N, Bucur PO, Ciacio O, Pittau G, Sa Cunha A, Adam R, Castaing D, Antonini T, Coilly A, Samuel D, Cherqui D, Vibert E. A new definition of sarcopenia in patients with cirrhosis undergoing liver transplantation. Liver Transpl 2017;23:143-54.

27. Streja E, Molnar MZ, Kovesdy CP, Bunnapradist S, Jing J, Nissenson AR, Mucsi I, Danovitch GM, Kalantar-Zadeh K. Associations of pretransplant weight and muscle mass with mortality in renal transplant recipients. Clin J Am Soc Nephrol 2011;6:1463-73.

28. Giraudo C, Cavaliere A, Lupi A, Guglielmi G, Quaia E. Established paths and new avenues: a review of the main radiological techniques for investigating sarcopenia. Quant Imaging Med Surg 2020;10:1602-13.

29. Englesbe MJ, Lee JS, He K, Fan L, Schaubel DE, Sheetz KH, Harbaugh CM, Holcombe SA, Campbell DA Jr, Sonnenday CJ, Wang SC. Analytic morphomics, core muscle size, and surgical outcomes. Ann Surg
2012;256:255-61.

30. Cahill GF Jr. Starvation in man. N Engl J Med 1970;282:668-75.

31. Biolo G, Fleming RY, Maggi SP, Nguyen TT, Herndon DN, Wolfe RR. Inverse regulation of protein turnover and amino acid transport in skeletal muscle of hypercatabolic patients. J Clin Endocrinol Metab 2002;87:3378-84.

32. Wolfe RR, Martini WZ. Changes in intermediary metabolism in severe surgical illness. World J Surg 2000;24:639-47.

33. Lam TK. Hepatic glucose metabolism in 2015: Nutrient and hormone-sensing-dependent regulation. Nat Rev Endocrinol 2016;12:70-2.

34. Hanai T, Shiraki M, Nishimura K, Ohnishi S, Imai K, Suetsugu A, Takai K, Shimizu M, Moriwaki H. Sarcopenia impairs prognosis of patients with liver cirrhosis. Nutrition 2015;31:193-9.

35. Grąt K, Pacho R, Grąt M, Krawczyk M, Zieniewicz K, Rowiński O. Impact of Body Composition on the Risk of Hepatocellular Carcinoma Recurrence After Liver Transplantation. J Clin Med 2019;8:1672.

36. Pedersen BK, Febbraio MA. Muscles, exercise and obesity: skeletal muscle as a secretory organ. Nat Rev Endocrinol 2012;8:457-65.

37. Ohishi W, Cologne JB, Fujiwara S, Suzuki G, Hayashi T, Niwa Y, Akahoshi M, Ueda K, Tsuge M, Chayama K. Serum interleukin-6 associated with hepatocellular carcinoma risk: a nested case-control study. Int J Cancer 2014;134:154-63.

38. Bouzakri K, Plomgaard P, Berney T, Donath MY, Pedersen BK, Halban PA. Bimodal effect on pancreatic $\beta$-cells of secretory products from normal or insulin-resistant human skeletal muscle. Diabetes 2011;60:1111-21.

39. Kamo N, Kaido T, Hamaguchi Y, Okumura S, Kobayashi A, Shirai H, Yao S, Yagi S, Uemoto S. Impact of sarcopenic obesity on outcomes in patients undergoing living donor liver transplantation. Clin Nutr 2019;38:2202-9.

Cite this article as: Tan Y, Duan T, Li B, Zhang B, Zhu Y, Yan K, Song J, Lv T, Yang J, Jiang L, Yang J, Wen T, Yan L. Sarcopenia defined by psoas muscle index independently predicts long-term survival after living donor liver transplantation in male recipients. Quant Imaging Med Surg 2022;12(1):215-228. doi: 10.21037/qims-21-314 